\title{
Traducción al castellano y validación de la escala Abbey para la detección del dolor en pacientes no comunicativos
}

\author{
P. Chamorro ${ }^{1}$ y E. Puche ${ }^{2}$ \\ ${ }^{1}$ Médico Geriatra. Hospital Cruz Roja. Almería. ${ }^{2}$ Prof. Farmacología Médica y Clínica. Facultad Medicina, \\ Universidad de Granada. Unidad de Farmacología Clínica. Hospital Universitario San Cecilio. Granada
}

Chamorro $P$, Puche E. Traducción al castellano y validación de la escala Abbey para la detección del dolor en pacientes no comunicativos. Rev Soc Esp Dolor 2013; 20(1): 3-7.

\footnotetext{
ABSTRACT

Objective: The validation of a pain assessment scale for non communicative Spanish patients: Spanish version of the Abbey scale.

Patients and methods: Geriatric unit in the hospital setting included all non-communicative patients over 60 years, with the exclusion of those who had respiratory failure, cerebral coma, severe brain trauma, or taking palliative oncological treatment. Variables: age, sex, carer, reason for admission, diagnosis, treatment, scale scores. Patients were evaluated using the Abbey scale for the detection of pain, Mini-Mental for evaluation of cognitive impairment, and the Global Deterioration Scale. When pain was detected it was treated with round the clock analgesics, and the patient was evaluated at 24 hour intervals, until pain was controlled, scale scores were noted each time.

Statistical Analyses: Descriptive study of the variables tested.

Psychometric properties: Reliability: Intrarater (ICC- intraclass correlation coefficient), Inter-rater (Kappa) and the internal consistency of the scale was measured using $\alpha$-Cronbach. Validity: predictive validity was measured using ROC curve analysis, concurrent validity (Spearman) and sensitivity to change (Wilcoxon).

Results: Data was collected from 119 non communicative patients; 71 (59.7\%) women, mean age $81.62 \pm 0.716$. Pain was detected in 44 patients (37.8\%). Reliability: ICC (intrarater $)=0.77$; kappa (inter-rater) $=0.65$ and $\alpha$-cronbach (internal
}

Recibido: 27-02-12.

Aceptado: 01-06-12. consistency) $=0.71$. Validity: concurrent validity with the holistic impression of the examiner Pearson coefficient $(r=0.82$, $\mathrm{p}=0.001$ ). Predictive Validity (ROC curve analysis) for a cut off score of 3; Specifity 100\% and sensitivity 95.6\% (Youden's Index 0.96). Sensitivity to change was of statistical significance $\mathrm{Z}=5.35$.

Conclusions: Although results were moderate, findings show evidence of reliability and validity of the Spanish version of the Abbey scale.

Key words: Pain non-communicative. Dementia. Observational scales. Abbey scale.

\section{RESUMEN}

Objetivo: El dolor en pacientes no comunicativos es un problema clínico a resolver. En nuestro país, no disponemos de momento, de un método validado al castellano para paliar el problema, por lo que nos propusimos hacerlo validando la escala Abbey.

Pacientes y métodos: Incluimos a aquellos pacientes mayores de 60 años, no comunicativos, de ambos sexos, y excluimos a aquellos con un estado febril o con diagnóstico de cáncer, traumatismo cráneo-encefálico, coma neurológico y a todo paciente que podía responder verbalmente a la pregunta de si "tiene usted dolor", que ingresaron a lo largo de un año en la unidad geriátrica de un hospital general. Al ingreso se aplicaron el MiniMental, la escala Reisburg (Global Deterioration Scale) y la versión española de la escala Abbey, a cada paciente que cumplía los criterios de inclusión. En aquellos pacientes con un dolor potencial, se hizo un seguimiento cada 24 h tras aplicar analgésicos con pauta horaria, según la escalera analgésica de la OMS, hasta la remisión del dolor.

Tratamiento estadístico: Propiedades psicométricos de fiabilidad; alfa-cronbach, concordancia interobservador (I:Kappa) e intraobservador (ICC). Propiedades psicométricos de validez; validez predictiva para un punto de corte de tres (curva ROC, especificidad y sensibilidad, I.Youden); validez concordante en- 
tre las puntuaciones de la escala y la impresión holística del investigador (coeficiente de correlación de Spearman), y la sensibilidad al cambio (test Wilcoxon). Estudio descriptivo de las variables de la muestra.

Resultados: Se recogieron datos de un total de 119 pacientes no verbales de ambos sexos con una edad de $80 \pm 0,7$ años; el $48 \%$ procedía de residencia y un $75 \%$ sufría demencia avanzada. El 36\% de los pacientes al ingreso tomaban analgésicos. Tras aplicar la escala detectamos 44 pacientes con un dolor potencial que fue resuelto con la pauta analgésica. Los valores para la consistencia interna ( $\alpha$ Cronbach $=0,71$ ); concordancia intraobservador (ICC $=0,77$ ) e interobservador (i. kappa $=0,65)$; sensibilidad al cambio $(Z=5,35)$; y valor predictivo (curva $\mathrm{ROC}=0,94$ ) fueron satisfactorios. La correlación entre la impresión holística del observador y la escala fue altamente significativa $(r=0,78, p<0,001)$.

Conclusión: A la vista de los aceptables resultados obtenidos proponemos a la escala Abbey en versión al castellano como método fácil, sencillo y confiable para detectar dolor en pacientes españoles no comunicativos.

Palabras clave: Pacientes no comunicativos. Dolor. Escalas, Abbey.

\section{INTRODUCCIÓN}

El dolor es el síntoma más frecuente en la práctica médica tanto hospitalaria como ambulatoria. El dolor se ha definido como una experiencia compleja multidimensional subjetiva, desagradable y emocional, asociada a una lesión tisular que todos experimentamos a lo largo de nuestra vida y que tiene una mayor prevalencia entre las personas ancianas (1-4).

Se ha descrito que el dolor crónico persistente produce en los pacientes graves repercusiones en su estado general, afectando a la esfera psíquica como física y con el consiguiente deterioro progresivo de la calidad de vida de los mismos $(5,6)$.

En la población anciana se da la doble situación de, por un lado es el sector poblacional con más alta prevalencia de dolor crónico, y por otro donde se dan un mayor número de diagnósticos que cursan con importantes alteraciones cognitivas, conducentes a estados de falta de comunicación, caso de la demencia avanzada, entre otros $(7,8)$.

Numerosos estudios epidemiológicos han demostrado que aquellos pacientes diagnosticados de una demencia avanzada, y por tanto no comunicativos, tienen mayores posibilidades de no ser diagnosticado ni tratado adecuadamente el dolor, se hallen o no institucionalizados $(1,4,7,9,10)$.

El diagnóstico del dolor requiere la colaboración directa del paciente sin la cual no es posible establecer sus características de manera precisa, por tanto es un problema clínico de difícil solución en aquellas personas en las que existe la imposibilidad para comunicarse.
A falta de un test que permita realizar de manera precisa y objetiva el diagnóstico de dolor, se han diseñado para estos pacientes no verbales una serie de escalas observacionales que intentan paliar la dramática situación que se le presenta al médico y a la enfermería con estos enfermos.

La SAG (Sociedad Americana de Geriatría) publicó en el 2002 una guía con una serie de indicadores de dolor, consensuados previamente por un panel de expertos y distribuidos en seis dominios relacionados con cambios de conducta, físicos y psíquicos en pacientes no comunicativos; dicha guía es actualmente un referente a la hora de diseñar un método capaz de detectar dolor en pacientes no verbales (11).

En nuestro país, de momento no tenemos un método para detectar dolor en pacientes no verbales pues las escalas disponibles en la bibliografía no están validadas ni traducidas al castellano, o las existentes han sido diseñadas para situaciones diferentes a las planteadas en este ensayo $(8,12)$. De ahí, que nos planteáramos escoger una escala entre las publicadas para pacientes no verbales que se ajustara a nuestro objetivo, que fuese fácil de aplicar, y que estuviese validada internacionalmente con un aceptable grado de fiabilidad.

Tras una amplia revisión en el MEDLINE (13-15) y basándonos en los criterios mencionados, escogimos la escala Abbey (16) con la intención de aportar un método fiable, rápido y fácil de aplicar a la cabecera del enfermo, por médicos y enfermeros, para la detección del dolor en pacientes no comunicativos.

\section{PACIENTES Y MÉTODOS}

El estudio se llevó a cabo durante doce meses (enerodiciembre 2009) en pacientes no comunicativos que ingresaron en la unidad geriátrica del complejo hospitalario Torrecárdenas (Almería). Se recogieron los datos de la historia clínica referentes a edad, sexo, domicilio, diagnóstico de admisión, co-morbilidad y tratamiento recibido. A los pacientes incluidos en el ensayo se les aplicó de entrada el Mini mental y la escala GDS (Global Deterioration scale of Reisburg) para valorar su estado cognitivo y funcional, y a continuación la escala Abbey, traducida al castellano previamente. En aquellos pacientes en los que se detectó dolor se les prescribieron analgésicos con pauta horaria, según criterios OMS, volviendo a valorar a dichos enfermos cada 24 h hasta control del síntoma. Se hizo un seguimiento a cincuenta pacientes sin dolor a las $24 \mathrm{~h}$ de la valoración inicial, para así poder medir la estabilidad temporal de la escala. Toda la información fue recogida por una misma persona en un cuestionario construido ad hoc siendo posteriormente transferida, previa depuración de errores, a una base de datos para el análisis estadístico con el programa SPSS 17. 
TABLA I. ESCALA ABBEY EN VERSIÓN AL CASTELLANO

\begin{tabular}{ll}
\hline Vocalización & $\begin{array}{l}\text { gimoteo, gemidos, llanto, } \\
\text { gritos }\end{array}$ \\
Expresión facial & $\begin{array}{l}\text { tensa, ceño fruncido, muecas, } \\
\text { expresión de miedo } \\
\text { inquietud, mecerse, proteger } \\
\text { una zona, retraimiento }\end{array}$ \\
Cambio conducta & $\begin{array}{l}\text { confusión, agitación, negativa } \\
\text { a la ingesta }\end{array}$ \\
Cambios fisiológicos & $\begin{array}{l}\text { temperatura, pulso, tensión } \\
\text { arterial }\end{array}$ \\
Cambios físicos & $\begin{array}{l}\text { erosiones, lesiones previas, } \\
\text { áreas de presión, deformidades } \\
\text { osteoarticulares, artritis, } \\
\text { contracturas }\end{array}$ \\
\hline
\end{tabular}

Cada item se valora como: ausente $=0$; leve $=1$; moderado $=2$; importante $=3$

Interpretación de la escala: no dolor $=0-3$; dolor leve $=3-7$; moderado $=8-13 ;$ grave $>14$

Como criterios de inclusión, todos los pacientes de ambos sexos mayores de 60 años no comunicativos que ingresaron en la unidad geriátrica del hospital a lo largo de doce meses por causas diferentes. Como criterios de exclusión, los pacientes menores de 60 años, y aquellos otros mayores de sesenta años con fiebre, insuficiencia respiratoria, traumatismo cráneo-encefálico severo, coma neurológico y cáncer con tratamiento.

Para la realización del estudio se obtuvieron los permisos oportunos de la comisión de ética e investigación del hospital, el consentimiento informado de familiares, comprometiéndose los autores a mantener en todo momento la confidencialidad de datos de los pacientes.

\section{Escala Abbey}

Es una escala observacional construida para evaluar el dolor en pacientes con demencia avanzada e internacionalmente validada (16-18) que cubre cuatro de los seis dominios descritos por la SAG y consta de seis items: vocalización, expresión facial, lenguaje corporal, cambios de comportamiento, cambios fisiológicos y cambios físicos (Tabla I). Una vez traducida al castellano se hizo la traducción inversa por un traductor certificado y fue presentada a un comité de expertos formado por seis médicos geriatras, un médico de la unidad del dolor y un internista que hicieron sus observaciones, al respecto. Esta escala además intenta, de forma rápida, establecer la intensidad
ANEXO I. ESCALA DE DOLOR ABBEY

Vocalización: lamentos, gruñidos, llanto

Ausente 0 Leve 1 Moderado 2 Grave 3

Expresión facial: expresión tensa, fruncida, lamentándose, aspecto asustado

Ausente 0 Leve 1 Moderado 2 Grave 3

Cambios de lenguaje corporal: movimientos de nerviosismo, de vaivén, protegiendo una parte del cuerpo, retraido.

Ausente 0 Leve $1 \quad$ Moderado 2 Grave 3

Cambios de comportamiento: aumento de confusión, rehúsa comer, alteración de patrones usuales

Ausente 0 Leve 1 Moderado 2 Grave 3

Cambios fisiológicos: temperatura, pulso o de tensión sanguínea fuera de los límites normales, sudor, enrojecimiento facial o palidez

Ausente 0 Leve $1 \quad$ Moderado 2 Grave 3

Cambios físicos: cortes en la piel, áreas de presión, artritis, contracturas, heridas anteriores

\begin{tabular}{lrrr} 
Ausente 0 & Leve 1 & Moderado 2 & Grave 3 \\
\hline Suma Puntuación Total & & \\
0-2 Sin dolor & 3-7 Leve & 8-13 Moderado & 14+ Severo
\end{tabular}

del dolor en leve, moderado y grave según la puntuación final alcanzada (Anexo 1).

\section{Diseño del estudio}

Se planificó un estudio prospectivo en pacientes no comunicativos a los que se les aplicó la escala Abbey al ingreso para detectar dolor. En aquellos pacientes con puntuación positiva (punto de corte 3 puntos) se instauró tratamiento analgésico siempre con pauta horaria y según criterios OMS. Se re-evaluó al paciente cada 24 horas hasta control del síntoma, anotándose las puntuaciones alcanzadas con la escala en cada evaluación y los analgésicos prescritos antes y después del tratamiento.

\section{Propiedades psicométricas y tratamiento estadístico}

En cuanto al tratamiento estadístico, se hizo en primer lugar una descriptiva de las diferentes variables recogidas y posteriormente una tabla crosstabs para calcular las posibles diferencias entre dos grupos de pacientes, con y sin dolor, con y sin demencia. En todos los casos se estableció el límite de significación estadística en $\mathrm{p}$ $<0,05$. 
TABLA II. DESCRIPCIÓN GENERAL DE LA MUESTRA

\begin{tabular}{ll}
\hline Total pacientes & 119 \\
Edad (media \pm DS) años & $81 \pm 0,7$ \\
Mujeres & $71 \quad 59,7 \%$ \\
Procedían residencia & $57 \quad 48 \%$ \\
Con demencia & $8975 \%$ \\
Tratamiento analgésico previo & 36 \\
Dolor potencial escala Abbey & $44 \quad 37 \%$ \\
\hline
\end{tabular}

Para medir la fiabilidad se calculó la consistencia interna (Cronbach), la concordancia interobservador (i. kappa) e intraobservador (ICC). La validez predictiva con la curva ROC, para un punto de corte de 3 en cuanto a presencia de dolor, relacionando la sensibilidad y la especificidad a través del índice de Youden. Para la sensibilidad al cambio el valor Z (test de Wilcoxon), comparando las puntuaciones de la escala antes y después del tratamiento.

Para la validez concurrente se correlacionó la impresión holística de dolor por el observador, usando una escala de valoración numérica (EVN) frente al resultado obtenido con la escala Abbey mediante el test de Spearman.

\section{RESULTADOS}

Se escogieron un total de 119 pacientes no comunicativos de ambos sexos, con un promedio de edad de $81 \pm$ 0,7 años (media \pm DS), de los que un $43 \%$ estaban institucionalizados y el $75 \%$ tenían una demencia previa (57 presentaron una enfermedad de Alzheimer y 32 una demencia vascular). (Tabla II). Las tres causas mayores de ingreso hospitalario fueron el $\mathrm{ACV}$ isquémico o hemorrágico $(30 \%)$, infección respiratoria $(15 \%)$ e infección urinaria (8\%). De todos los pacientes, el 36\% tomaba analgésicos a su ingreso. Cuando se aplicó el Mini Mental obtuvimos un valor de cero y con la escala GDS entre 6-7 puntos, lo que se correspondió con una muestra de pacientes con gran incapacidad y deterioro cognitivo importante. Tras aplicar la escala Abbey a todos los enfermo, hallamos 44 pacientes (37\%) con un cuadro doloroso, siendo posible establecer las causas y localización del dolor en el $21 \%$ de los mismos, destacando las úlceras de presión, las contracturas musculares y las deformidades en huesos y articulaciones por enfermedad degenerativa (artrosis). La puntuación media de la escala para los pacientes con dolor fue de 3,9 $\pm 1,1$ frente al $0,8 \pm 0,8$ sin dolor ( $\mathrm{p}=0,001)$. No se hallaron diferencias estadísticamente significativas para la edad, sexo o procedencia en cuanto a la puntuación de la escala.

Los test psicométricos dieron los siguientes valores: una consistencia interna con un alfa de Cronbach $=0,71$; una concordancia intraobservador ICC $=0,77(0,59-0,86$ IC 95\%) e interobservador i. kappa $=0,65(\mathrm{p}=0,001)$; una sensibilidad al cambio con un valor $\mathrm{Z}=5,35(\mathrm{p}=$ $0,001)$; un valor predictivo positivo del $100 \%$ (ROC $=$ $0,94)$; una especificidad del $100 \%$ y una sensibilidad del $96 \%$ (i. Youden $=0,96$ ). La validez concurrente entre la impresión holística del observador y la escala Abbey, fue estadísticamente significativa tras aplicar el test de Pearson $(\mathrm{r}=0,82, \mathrm{p}=0,001)$.

De los cuarenta y cuatro pacientes en los que detectamos un dolor potencial y tras iniciar un tratamiento analgésico con pauta horaria observamos que a las $72 \mathrm{~h}$ ningún paciente presentaba dolor. De todos los pacientes con un dolor potencial, treinta y siete tenían una demencia avanzada con un mayor porcentaje para el tipo Alzheimer (74\%). En las puntuaciones de la escala no hallamos diferencias entre pacientes con y sin demencia en cuanto a la detección de dolor tras aplicar la escala.

\section{DISCUSIÓN}

La detección del dolor, nociceptivo o neuropático, persistente en personas no comunicativas es un problema clínico por resolver. Para ello se han construido escalas observacionales más o menos complejas que intentan remediar el problema, ninguna validada al castellano hasta ahora.

Aunque los resultados obtenidos por todas las escalas en los estudios realizados, incluida la Abbey, son considerados moderados y con algunos aspectos discutibles, como la sospecha de que solo detectan dolor en una parte de los pacientes, no discriminan el tipo de dolor, y si los cambios de conducta observados en los pacientes son indicadores fehacientes de la presencia de dolor, entre otros (13), de momento no disponemos de otro método fiable y objetivo para resolver la dramática situación que se le presenta al médico y enfermería en este tipo de pacientes no verbales, respecto del control de su dolor y sufrimiento.

La escala Abbey aunque comparte las limitaciones ya comentadas para todo método similar, sin embargo está catalogada como una herramienta fiable y útil para detectar dolor en pacientes no verbales (13-15) estando validada al inglés (16), japonés (17) e italiano (18).

Si bien la escala Abbey fue diseñada en un principio para pacientes con una demencia avanzada, pensamos que podría también aplicarse a pacientes no verbales por causas distintas a la demencia pero esto requiere confirmación.

La razón de una mayor presencia de pacientes con demencia en nuestro estudio se debió a que esta es una enfermedad muy prevalente entre los mayores, lo cual concuerda con los datos de la bibliografía consultada $(19,20)$.

De otro lado, la buena correlación obtenida entre la escala Abbey y la impresión holística del observador, un 
médico con amplia experiencia clínica, creemos que es una prueba más a favor de la utilidad de esta escala para detectar el dolor en este tipo de pacientes. De momento no disponemos en pacientes españoles de otro método de referencia que nos sirva para validar comparativamente la escala Abbey traducida al castellano.

Concluimos diciendo que el diagnóstico del dolor en pacientes no comunicativos tiene un elevado grado de dificultad siendo el uso de escalas observacionales el único método, por ahora, capaz de paliar con todas sus limitaciones esta dramática situación. De acuerdo a nuestros resultados proponemos a la escala Abbey traducida al castellano como un método confiable para la detección del dolor en pacientes no comunicativos; esta no requiere una compleja preparación para aplicarse y se cumplimenta en poco más de un minuto a la cabecera del paciente.

A pesar de los buenos resultados obtenidos creemos que se requieren estudios más amplios con esta escala, aplicada a pacientes españoles no verbales de larga estancia, con y sin demencia, que confirmen los hallazgos de este ensayo.

\section{Agradecimientos}

D. Pablo Garrido Fernández, bioestadístico, FIBAO, CHT Almería. Dra. Emilia López Lirola, Geriatra, por su ánimo constante, CHT Almería.

\section{CORRESPONDENCIA}

Pilar Chamorro

Teléfono: 669769725

e-mail: pchamorro@hotmail.com

\section{BIBLIOGRAFÍA}

1. Ferrell BA, Ferrell BR, Osterweil D. Pain in nursing home. JAGS 1990;38:409-14.

2. Gagliese L, Melzack R. Chronic pain in elderly people. Pain 1997;70:3-14.
3. Mellar D, Manish S. Demographics, assessment and management of pain in the elderly. Drug Aging 2003;20:23-57.

4. Alaba J, Arriola E. Prevalencia de dolor en pacientes geriátricos institucionalizados. Rev Soc Esp Dolor 2009;16:344-52.

5. Epps CD. Recognizing pain in the institutionalized older with dementia. Geriatr Nurs 2001;22:71-7.

6. Katz N. The impact of pain management on quality of life. J Pain Symtom Manege 2002;24:S38-S47.

7. Ferrell BA. Pain management in elderly people. J Am Geriatr Soc 1991;39:64-73.

8. Alaba J, Arriola E, Navarro A, et al. Demencia y dolor. Rev Soc Esp Dolor 2011;18:176-86.

9. Nikolaus T, Zeyfang A. Pharmacological treatments for persistent non-malignant pain in older persons. Drug Aging 2001;21:19-41.

10. Won A, Lapane K, Vallow S, et al. Persistent non-malignant pain analgesia prescribing pattern in elderly nursing home residents. J Am Geriatr Soc 2004;52:867-74.

11. AGS panel on persistent pain in older persons. J Am Geriatr Soc 2002;50(Supl 6);S205-24.

12. Latorre M, Solís M, Falero, et al. Validación de la escala de conductas indicadoras de dolor para valorar el dolor en pacientes críticos no comunicativos y sometidos a ventilación mecánica: resultados proyecto ESCID. Enf Intens 2011;22:3-12.

13. Herr K, Bjoro K, Decker D. Tools for assessment pain in nonverbal older adults with dementia. J Pain Symtom Manege 2006;31:170-92.

14. Zwakhalen S, Hamers J, Huijer H, Berger M. Pain in elderly people with severe dementia: a systematic review of behavioural pain assessment tools. BMC Geriatrics 2006;6:3-18.

15. Aubin M, Ciguere A, Hadjistavropoulos T, Verrault R. L'evaluation systematique des instruments pour mesurer la douleur chez les personnes âgées ayant des capacités réduites á communiquer. Pain Res Manege 2007;12:195-203.

16. Abbey J, Piller N, Bellis de A, et al. The Abbey pain scale: a 1 minute numerical indicator for people with end stage dementia. Int J Palliat Nurs 2004;10:6-14.

17. Takai Y, Yamamoto N, Chiba Y, et al. Abbey scale pain: development and validation of the japanese version. Geriatr Gerontol Inter 2010;10:145-53.

18. Storti M. The validation of a pain assessment scale for patients with cognitive impairment: the Italian version of Abbey scale. Recenti Prog Med 2009;100:405-9.

19. Bermejo F, Benito J, Vega S, et al. Incidence and subtypes of dementia in three elderly populations of central Spain. J Neurol Sci 2008;264:63-72.

20. Di Carlo A, Baldereschi M, Amaducci L, et al. Incidence of dementia, Azheimer's disease, and vascular dementia in Italy. The ILSA study. J Am Geriatr Soc 2002;50:41-8. 